\title{
Evaluation of the bacterial diversity of Pressure ulcers using bTEFAP pyrosequencing
}

\author{
Drake M Smith', David E Snow ${ }^{1}$, Eric Rees, Ann M Zischkau', J Delton Hanson', Randall D Wolcott ${ }^{2}$, Yan Sun ${ }^{1}$, \\ Jennifer White', Shashi Kumar ${ }^{1}$, Scot E Dowd ${ }^{1,3^{*}}$
}

\begin{abstract}
Background: Decubitus ulcers, also known as bedsores or pressure ulcers, affect millions of hospitalized patients each year. The microflora of chronic wounds such as ulcers most commonly exist in the biofilm phenotype and have been known to significantly impair normal healing trajectories.

Methods: Bacterial tag-encoded FLX amplicon pyrosequencing (bTEFAP), a universal bacterial identification method, was used to identify bacterial populations in 49 decubitus ulcers. Diversity estimators were utilized and wound community compositions analyzed in relation to metadata such as Age, race, gender, and comorbidities.

Results: Decubitus ulcers are shown to be polymicrobial in nature with no single bacterium exclusively colonizing the wounds. The microbial community among such ulcers is highly variable. While there are between 3 and 10 primary populations in each wound there can be hundreds of different species present many of which are in trace amounts. There is no clearly significant differences in the microbial ecology of decubitus ulcer in relation to metadata except when considering diabetes. The microbial populations and composition in the decubitus ulcers of diabetics may be significantly different from the communities in non-diabetics.

Conclusions: Based upon the continued elucidation of chronic wound bioburdens as polymicrobial infections, it is recommended that, in addition to traditional biofilm-based wound care strategies, an antimicrobial/antibiofilm treatment program can be tailored to each patient's respective wound microflora.
\end{abstract}

\section{Background}

An estimated 2.5 million hospitalized Americans currently suffer from decubitus ulcers, also known as bedsores or pressure ulcers,[1] and the annual cost for the prevention and treatment of decubitus ulcers is approximately $\$ 10$ billion [2]. Chronic wounds such as ulcers have troubled the medical community for centuries, as the first known decubitus ulcer was detected during the autopsy of an Egyptian mummy [3]. Today, approximately $20 \%$ of long-term care patients are affected [2].

Decubitus ulcers often result from both external and internal patient factors [4]. Pressure, friction, shear force, and moisture are controllable external factors that affect a patient's susceptibility to decubitus ulcers [5-7]. Internal patient factors such as fever, malnutrition, endothelial dysfunction, and anemia also contribute to a

\footnotetext{
* Correspondence: sdowd@pathogenresearch.org

${ }^{1}$ Research and Testing Laboratory, Lubbock, TX 79407, USA

Full list of author information is available at the end of the article
}

patients susceptibility to ulceration $[6,8]$. Although exceptional care may alleviate external ulcer promoting factors, skin integrity is largely dependent on overall patient health. The dermis and epidermis rely on other organ systems for nutrition and immune function which when compromised by poor health considerably increase the risk of ulceration [9]. As reviewed elsewhere, those typically most at risk of developing decubitus ulcers are the aged, debilitated, paralyzed, unconscious, or patients with incontinentia pigmenti $[4,6,10-12]$. Less typical, though of equal concern, are children with similar compromising health conditions [13-15].

Decubitus ulcers are most commonly found on the lower half of the body along bony prominences such as the sacrum and heels of bedridden patients $[4,16]$. Blood flow to compressed tissue becomes restricted and over time nutrient distribution comes to a relative halt while toxic metabolites begin to accumulate, causing cell death [4,17-19]. Additionally, because circulation is restricted, the patient's immunological response in the 
vicinity of the wound becomes ineffective and the ability to heal is compromised [8].

The microbiota of chronic wounds is known to play a significant role in the hindrance of wound healing, even in the absence of inflammation. This microbial bioburden exists in wounds predominantly as a biofilm $[8,10,20-28]$. The onset of decubitus ulcers occurs as described, and it is logical to suggest that this impaired host environment is extremely susceptible to biofilm incursion [29]. Functionally equivalent pathogroups (FEPs) are symbiotic colonies of otherwise nonpathogenic species that act synergistically to promote their own survival at the expense of the host. These FEPs colonize and exist as a cohabitation of many bacterial species, known as a chronic wound pathogenic biofilm (CWPB). It is widely accepted that CWPBs are the primary infectious agent in chronic wounds [20,22,25-32].

Current research efforts have exposed CWPBs as being extremely resistant to antimicrobial therapy and as highly adaptable systems with complex ecologies $[21,33]$. The use of universal $16 \mathrm{~S}$ rRNA amplification and sequencing molecular methods, specifically bacterial tag-encoded FLX amplicon pyrosequencing (bTEFAP), have uncovered many FEPs which comprise CWPBs [22,23,33-39]. As the diversity of these CWPB communities increases, the effectiveness of the host's immune system seems to decrease, leading to the effects on wound healing discussed previously [32].

Several helpful reviews have recently been published summarizing efforts within the scientific community to better understand the microbiology associated with chronic wounds, the resistance these wounds develop as a function of CWPBs, and improvements in the effective use of antibiotics and other treatments on wound healing $[21,40]$. Also evident in the literature are recent efforts to survey the microbial diversity of venous leg ulcers [41]. However, other work indicates significant differences among pathogens in various wound types, and a survey of decubitus ulcers is a necessary contribution to this body of work [37]. Therefore, the aim of this work is to shed new light on the polymicrobial diversity of chronic decubitus ulcer biofilm infections using bacterial tag-encoded FLX amplicon pyrosequencing.

\section{Results}

The diversity of 49 individual decubitus ulcers was evaluated using the bTEFAP methodology. A total of 225,937 individual sequences longer than 350 bp were analyzed among the 49 samples with 210,836 sequences generating BLASTn hits against the bacterial database and an average sequence identity of $96.7 \%$. A total of 83,705 sequences $(39.7 \%)$ had identity below $96.5 \%$. Only 2,000 of the total number of analyzed sequences fell below $80 \%$ identity. A traceback analysis based upon the divergence of sequences from well described and type sequences was performed.

A total of 212 genera and 487 predicted species (occurring in at least 2 of the wounds) were identified among the 49 wounds. The top 25 unique and most ubiquitous genera (or closest taxonomic designation) are indicated in Table 1 and the top 25 species occurring in the wounds are shown in Table 2. Several genera and species were found in high percentage in individual wounds (Figure 1 and Figure 2). Corynebacterium striatum predominated in 12 of the wounds; Streptococcus agalactiae predominated in 9 wounds; Pseudomonas aeruginosa predominated in 6 wounds; Anaerococcus vaginalis and Anaerococcus prevotii predominated in 1 wound each, and a mixture of these two species with Anaerococcus lactolyticus led to a predominance of this genus in 3 additional wounds; Serratia marcescens predominated in 4 wounds; Staphylococcus aureus predominated in 3 wounds and a mixture of Staphylococcus piscifermentans and Staphylococcus epidermidis led to a predominance of this genus in a fourth wound; Enterococcus faecalis predominated in 3 wounds; Prevotella bivida predominated in 2 wounds and a mixture of Prevotella bivida with Prevotella buccalis led to a predominance of this genus in a third wound; Finegoldia magna, Fusobacterium nucleatum, and Porphyromonas somerae were predominant in 2 wounds each; and Bacteroides fragilis, Klebsiella pneumoniae, Acinetobacter baumannii, Curvibacter gracilis, and Proteus mirabilis were each predominant in 1 wound. The remaining wounds were highly diverse with no significantly predominant populations. From the data we note that $69 \%$ were gram positive, $31 \%$ were anaerobes, $43 \%$ were facultative anaerobes, and $69 \%$ were rod shaped bacteria (Figure 3). It should also be noted that genera appearing in less than 3 individual samples and also having only low relative percentages $<1 \%$ were omitted from this figure to improve readability.

We further evaluated microbial diversity in relation to metadata that included Age group, Gender, Race, diabetes, vascular or coronary co-morbidities, paraplegia or quadriplegia, and time it took to heal the wound. There were no significant difference or clustering of the microbial diversity observed in relation to any of the metadata except diabetes (Figure 4). Based upon the PCA1 loading and two tailed $t$-test the separation explained by the primary vector was significant for microbial diversity in the ulcers depending on whether the patient had diabetes $(p=0.003)$. Measures of diversity analyses were evaluated using Rarefaction, Shannon index, Ace, and Chao 1 at the $3 \%$ and $5 \%$ divergence levels (corresponding to species and genus respectively). An average of 3964 sequences per sample were evaluated with 
Table 1 Evaluation of primary genera among the 49 decubitus ulcer samples

\begin{tabular}{|c|c|c|c|c|c|c|c|}
\hline ID & No. of samples & Ave $\%$ & Std Dev & Max\% & Gram Stain* & Oxygen Tolerance & Morphology (shape) \\
\hline Streptococcus & 45 & 19.0 & 33.65 & 97.5 & + & Facultative anaerobe & Cocci \\
\hline Corynebacterium & 44 & 24.8 & 31.7 & 99.3 & + & Aerobe & Rod \\
\hline Staphylococcus & 39 & 9.2 & 25.0 & 99.7 & + & Facultative anaerobe & Cocci \\
\hline Finegoldia & 32 & 7.3 & 18.1 & 83.5 & + & Anaerobe & Cocci \\
\hline Anaerococcus & 29 & 6.6 & 9.1 & 36.0 & + & Anaerobe & Cocci \\
\hline Pseudomonas & 27 & 14.1 & 23.7 & 82.0 & - & Aerobe & Rod \\
\hline Peptoniphilus & 27 & 3.6 & 5.1 & 19.4 & + & Anaerobe & Cocci \\
\hline Enterococcus & 24 & 8.5 & 19.5 & 79.9 & + & Facultative anaerobe & Cocci \\
\hline Prevotella & 24 & 7.0 & 16.0 & 69.0 & - & Anaerobe & Rod \\
\hline Clostridium & 21 & 1.49 & 3.4 & 14.5 & + & Anaerobe & Rod \\
\hline Pelomonas & 18 & 1.5 & 3.4 & 11.1 & - & Aerobe & Rod \\
\hline Bacteroides & 17 & 7.5 & 24.0 & 99.9 & - & Anaerobe & Rod \\
\hline Flavobacterium & 17 & 2.8 & 7.4 & 30.8 & - & Aerobe & Bacillus or coccobacillus \\
\hline Porphyromonas & 16 & 3.4 & 7.5 & 23.5 & - & Anaerobe & Rod \\
\hline Serratia & 15 & 21.1 & 30.0 & 94.9 & - & Facultative anaerobe & Rod \\
\hline Escherichia & 15 & 2.5 & 4.14 & 12.2 & - & Facultative anaerobe & Bacillus or coccobacillus \\
\hline Brevibacterium & 14 & 2.2 & 3.1 & 10.8 & + & Aerobe & Rod \\
\hline Eubacterium & 14 & 1.3 & 2.1 & 6.2 & + & Anaerobe & Rod \\
\hline Arthrobacter & 14 & 0.3 & 0.3 & 1.0 & - & Aerobe & Rod \\
\hline Peptostreptococcus & 12 & 2.6 & 2.9 & 9.1 & + & Anaerobe & Coccus \\
\hline Helococcus & 12 & 1.3 & 2.7 & 9.8 & + & Facultative anaerobe & Cocci \\
\hline Fusobacterium & 11 & 9.6 & 19.4 & 63.9 & - & Anaerobe & Rod \\
\hline Dermabacter & 11 & 0.3 & 0.3 & 0.9 & + & Facultative anaerobe & Rod \\
\hline Sulfurospirillum & 10 & 1.0 & 2.0 & 6.2 & - & Aerobe & Rod \\
\hline Dialister & 10 & 0.6 & 1.0 & 3.2 & - & Anaerobe & Rod \\
\hline
\end{tabular}

The primary identification based upon percent sequence identity as described in the materials and methods is indicated. The number of samples in which each bacteria was identified is provided along with the average percent (avg \%) among the positive samples, the standard deviation (st dev) and the range of percentages among the positive samples. No. of samples = the no. of samples in which the genus were identified Mean $\%=$ the average percentage of that genus in each of the samples SD = standard deviation of these percentages Maximum $\%=$ the maximum percentage of the genus in each sample ${ }^{*}+$ Grampositive; - Gram-negative

† Anaerobes are unable to propogate in laboratory media in the presence of oxygen; facultative anaerobes can grow both in the presence and absence of oxygen; aerobes can grow in the presence of oxygen

summary data on average diversity measurements evaluated statistically using Mann-Whitney and KruskalWallis tests in relation to the metadata. No significant differences were observed for diversity measures in relation to metadata parameters even antibiotic usage.

\section{Discussion}

The results indicate that there was a considerably large diversity in the samples, with 228 identified genera and 487 identified species among 49 decubitus ulcers. Additionally, the 79,837 sequences with identity less than 96.5\% can be considered as previously unknown or uncharacterized species of bacteria [42]. The majority of these organisms were most closely related to Staphylococcus, Enterococcus, Serratia, Pseudomonas, Streptococcus, and Corynebacterium spp based upon $16 \mathrm{~S}$ sequence. These bacteria were classified based upon their closest identification and ranked at the most suitable genus, family or order level.

Our data indicates a number of important FEPs associated with decubitus ulcers (Figure 1 and Figure 2 dendograms). At a relative distance of 0.30 based upon the weighted-pair linkage and Manhattan distance in Figure 2 we note there are 8 primary clusters, which included 8 predominant groups representing possible pathogroups. It is also evident that Staphylococcus aureus, Corynebacterium striatum, Streptococcus agalactiae, Serratia marcescens, Finegoldia magna, Pseudomonas aeruginosa, and Enterococcus faecalis are defining variables for these 8 clusters, with Streptococcus agalactiae being the defining variable for two separate clusters. It is interesting that so many of these wounds were predominated by what are either facultative or obligate anaerobic bacteria with only very minor populations of aerobes (36\%). This 
Table 2 Evaluation of primary species among the 49 decubitus ulcer samples

\begin{tabular}{|c|c|c|c|c|c|c|c|}
\hline ID & No. of samples & Ave $\%$ & Std Dev & Max \% & Gram Stain & Oxygen Tolerance & Morphology (shape) \\
\hline Corynebacterium striatum & 35 & 26.1 & 32.1 & 99.3 & + & Facultative anaerobe & Bacillus or coccobacillus \\
\hline Finegoldia magna & 32 & 7.3 & 18.1 & 83.5 & + & Anaerobe & Coccus \\
\hline Staphylococcus epidermidis & 24 & 1.8 & 3.3 & 12.1 & + & Facultative anaerobe & Coccus \\
\hline Anaerococcus vaginalis & 23 & 4.3 & 5.6 & 22.8 & + & Anaerobe & Coccus \\
\hline Pseudomonas aeruginosa & 23 & 16.5 & 24.9 & 82.0 & - & Aerobe & Bacillus or coccobacillus \\
\hline Streptococcus mitis & 22 & 0.6 & 1.9 & 8.9 & + & Facultative anaerobe & Coccus \\
\hline Streptococcus parasanguinis & 21 & 0.2 & 0.2 & 0.8 & + & Facultative anaerobe & Coccus \\
\hline Enterococcus faecalis & 21 & 8.7 & 20.6 & 79.9 & + & Facultative anaerobe & Coccus \\
\hline Anaerococcus lactolyticus & 20 & 2.2 & 3.0 & 8.5 & + & Anaerobe & Coccus \\
\hline Peptoniphilus indolicus & 20 & 1.6 & 2.4 & 8.6 & + & Anaerobe & Coccus \\
\hline Streptococcus agalactiae & 19 & 37.7 & 41.6 & 97.2 & + & Facultative anaerobe & Coccus \\
\hline Peptoniphilus harei & 18 & 0.6 & 1.2 & 4.3 & + & Anaerobe & Coccus \\
\hline Pelomonas saccharophila & 18 & 1.5 & 3.4 & 11.1 & - & Aerobe & Rod \\
\hline Staphylococcus aureus & 17 & 13.7 & 33.2 & 99.7 & + & Facultative anaerobe & Coccus \\
\hline Peptoniphilus ivorii & 17 & 1.9 & 3.2 & 11.0 & + & Anaerobe & Coccus \\
\hline Streptococcus thermophilus & 16 & 0.3 & 0.5 & 2.0 & + & Facultative anaerobe & Coccus \\
\hline Streptococcus constellatus & 16 & 0.3 & 0.4 & 1.6 & + & Facultative anaerobe & Coccus \\
\hline Escherichia coli & 15 & 2.5 & 4.1 & 12.2 & - & Facultative anaerobe & Bacillus or coccobacillus \\
\hline Flavobacterium succinicans & 15 & 0.9 & 1.2 & 3.6 & - & Aerobe & Bacillus or coccobacillus \\
\hline Serratia marcescens & 15 & 21.1 & 29.9 & 94.9 & - & Facultative anaerobe & Bacillus or coccobacillus \\
\hline Corynebacterium tuberculostearicum & 14 & 4.4 & 8.7 & 27.9 & + & Facultative anaerobe & Bacillus or coccobacillus \\
\hline Peptoniphilus lacrimalis & 13 & 1.0 & 1.8 & 6.2 & + & Anaerobe & Coccus \\
\hline Porphyromonas somerae & 13 & 4.0 & 8.1 & 23.3 & - & Anaerobe & Bacillus or coccobacillus \\
\hline Prevotella buccalis & 12 & 3.4 & 4.6 & 12.7 & - & Anaerobe & Bacillus or coccobacillus \\
\hline Brevibacterium antiquum & 12 & 2.4 & 3.1 & 10.8 & + & Aerobic & Rod \\
\hline
\end{tabular}

The primary identification based upon percent sequence identity as described in the materials and methods is indicated. The number of samples in which each bacteria was identified is provided along with the average percent (avg \%) among the positive samples, the standard deviation (std dev) and the range of percentages among the positive samples. No. of samples $=$ the no. of samples in which the species were identified Mean $\%=$ the average percentage of that species in each of the samples SD = standard deviation of these percentages Maximum \% = the maximum percentage of the species in each sample * + Grampositive; - Gram-negative

† Anaerobes are unable to propagate in laboratory media in the presence of oxygen; facultative anaerobes can grow both in the presence and absence of oxygen; aerobes can grow in the presence of oxygen

suggests that such anaerobes might be contributing significantly to the etiology of chronic wound biofilm infections.

Most of the wounds we have evaluated contain relatively high overall numbers of bacteria $\left(>10^{5}\right.$ per $\mathrm{mg}$ debridement), based upon quantitative molecular methods so even ostensibly low percentages of individual species may potentially represent a large number of individual bacteria that have colonized or been recruited into and commenced propagating within decubitus ulcer biofilms even if we are considering populations that represent only $1 \%$ of the total. It is also important to note that, in addition to chronic wounds such as decubitus ulcers, healthy human skin is host to a diverse community of microbes.

Several recent surveys have shown that intra- and inter-personal diversity of the human skin microbiome is highly variable depending on gender, body regions, handedness, and time [43-46]. Skin microflora are found to fluctuate greatly in a short period of time, [43] women are shown to have a higher diversity than men, the dominant hand is shown to be more diverse than its counterpart, and skin regions are shown to be more similar in microbial composition with greater proximity [44]. In addition, the genera Corynebacterium, Propionibacteria, and Staphylococcus have been identified as primary constituents of healthy skin microflora, with Corynebacterium and Staphylococcus predominating the moist environments [45]. It is no surprise, then, to have detected Corynebacterium and Staphylococcus in 44 and 39 respective wounds out of 49 surveyed. These ubiquitous skin bacteria may sometimes be the primary, opportunistic pathogenic constituents of CWPBs, as seen in Figures 1 and 2. 


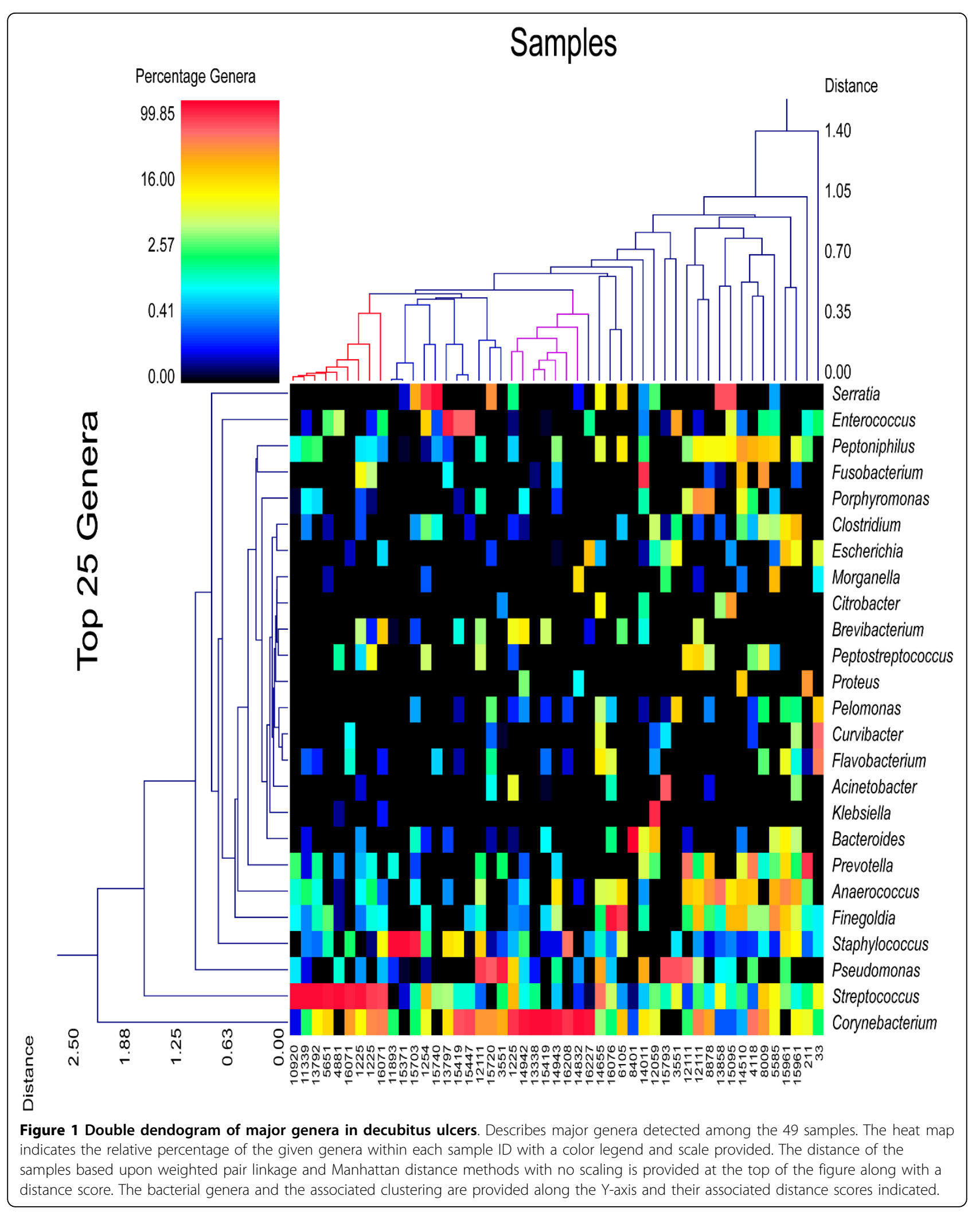




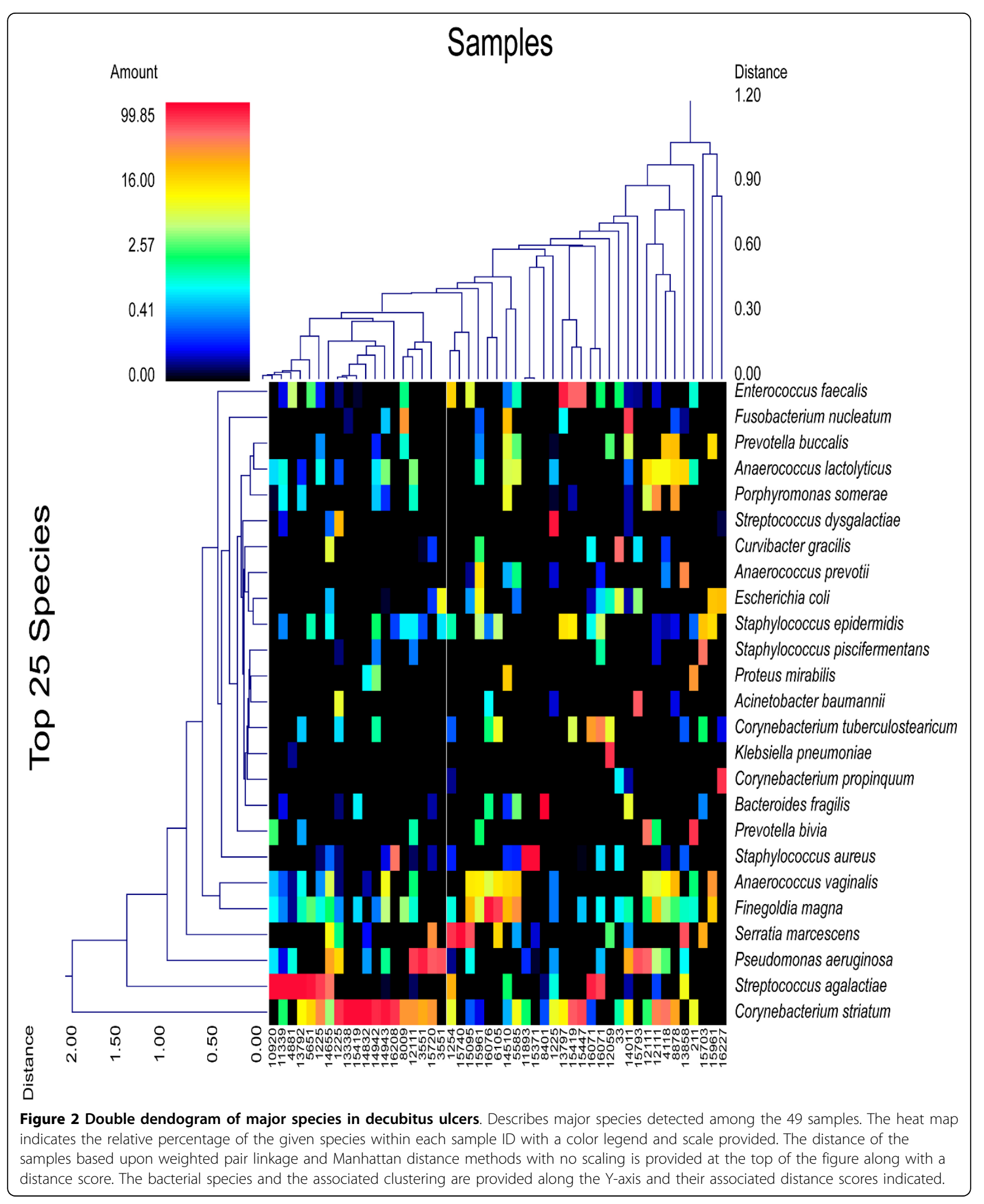




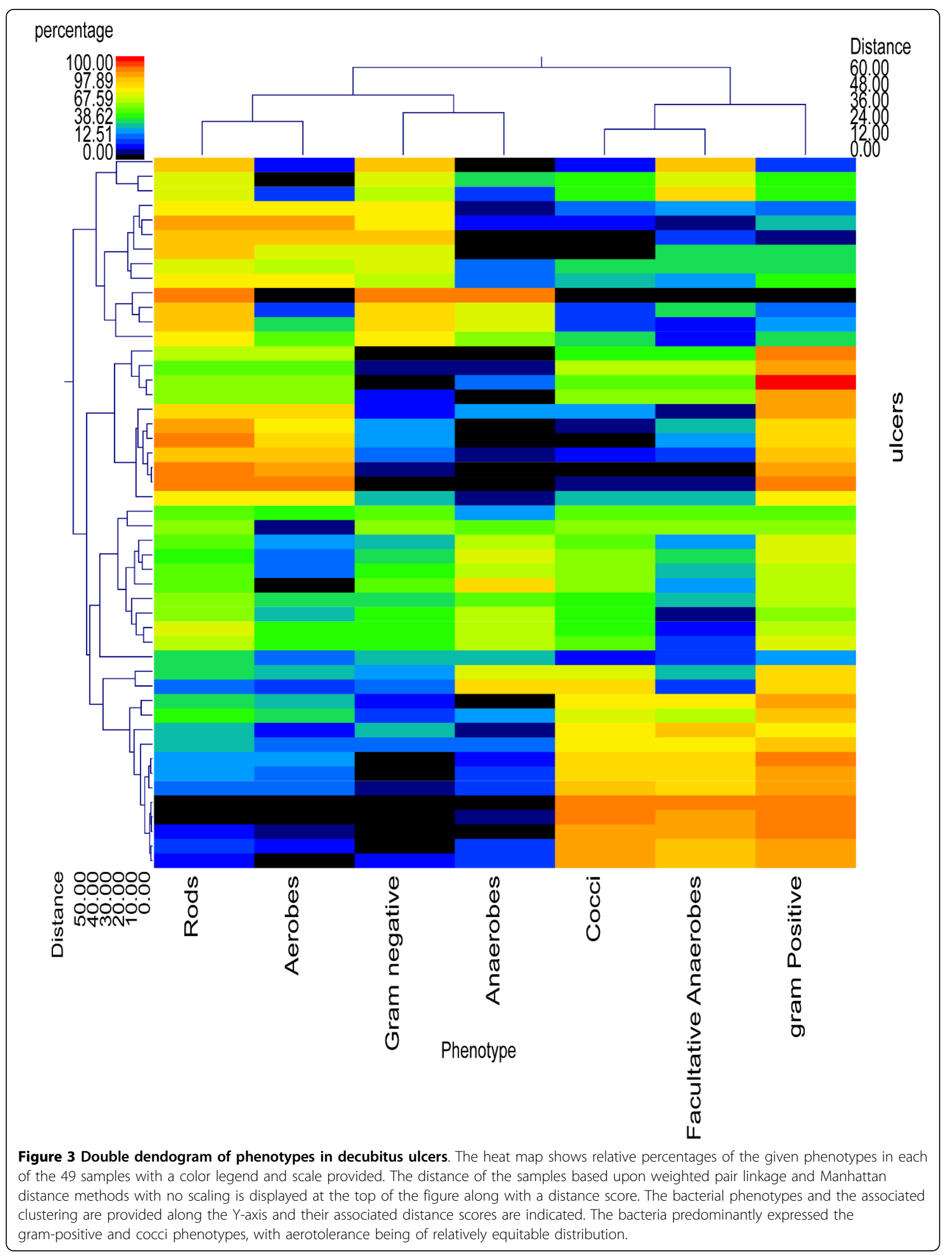




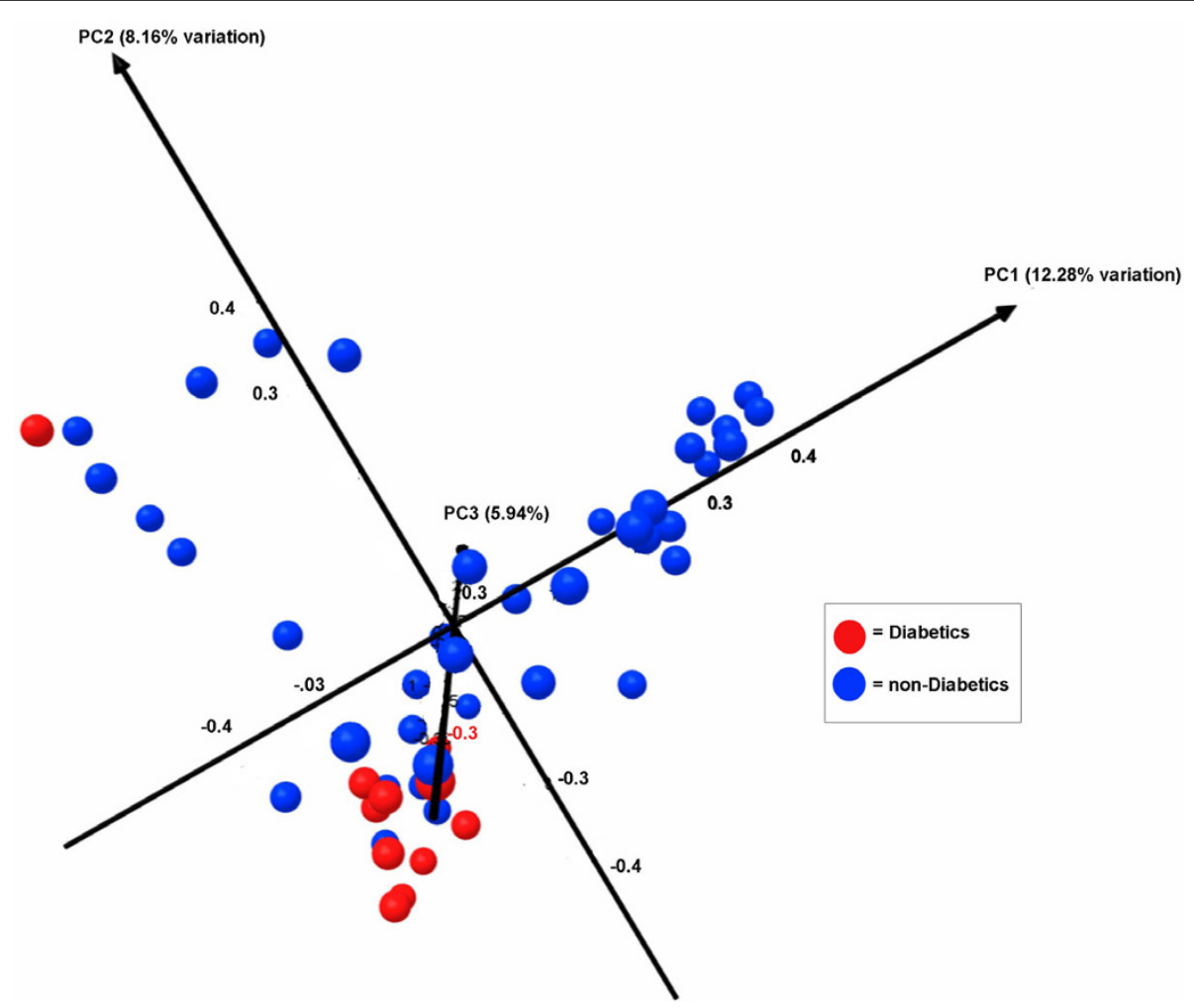

Figure 4 Three dimensional PCA plot of the unifrac distance in relation to diabetes. Principle component analysis based upon a unifrac analysis of the sequencing data was utilized. Based upon this PCA analysis the primary 3 vectors are plotted in 3 dimensions. The percent variability explained by each vector is indicated in parenthesis in the figure. Based upon the primary vector a t-test was utilized to determine if there was significant variation between diabetic samples. The separation across the primary vector was found to be significant $(p=0.003)$ indicating that the community structure of ulcers in subjects with diabetics may be different from those of non-diabetics.

Resident skin microflora may be considered largely commensal in nature, but it is known that nonpathogenic microbes often become opportunistically pathogenic when the skin barrier becomes impaired [47]. The difference between a pathogenic dermatophyte and a resident skin bacterium is commonly defined not only by the inherent properties of the organism, but also by the host's ability to resist infection. Resident skin bacteria are capable of expressing virulence factors which under certain circumstances may allow for the evasion of host defensive onslaught, but the primary determinant of pathogenicity lies in the effectiveness of host response to microbial invasion [48]. It may be that conditions within the open wound environment allow for much greater propagation of resident cutaneous microflora, which colonize the wound without substantial opposition. In some cases, these nonpathogenic skin residents may harbor pathogenic recruits by concealing antigenic factors, thus preventing recognition by the host's primary immune cells.

The 49 sampled wounds were found to be sufficiently diverse in composition as to negate any generalized recommendations for the targeting of microbial bioburdens within chronic wounds. Instead, each wound is shown to be exceptionally unique, with some wounds predominated by opportunistically pathogenic commensal skin residents and others predominated by known pathogens. Other wounds showed no clear predomination of a single species but rather a heterogeneous and complex assemblage. In addition, the present study is incapable of determining the role of certain bacteria that have been detected in lower, albeit potentially significant percentages. Even bacteria which comprise approximately $15 \%$ of a wound, as indicated in Figures 1 and 2, may contribute to the etiology of chronic wounds as this percentage represents a substantial number of bacteria even in a wound with only moderate bioburden at critical colonization levels. It is therefore recommended that future treatment courses be customized for each individual wound due to the highly variable etiology of chronic wounds such as decubitus ulcers.

Taking into consideration the metadata we did not find any significant differences in ecological measurements of microbial diversity or population richness (rarefaction, ace, Chao1) we did notice a significant difference in ulcers associated with their community 
composition in relation to whether the patient had diabetes or not. This suggests that the types of pathogens and opportunistic pathogens that populate pressure ulcers may be significantly different if the subject also has diabetes. Diversity measurements suggest that up to an average of 337 species and 180 genera of bacteria may be present in a pressure wound. These numbers can be misleading on two levels. First further optimization of next generation data processing will enhance the reliability of such measurements from a technical standpoint. Second, it is well understood that many of the bacteria that may be present in chronic wounds may not be active participants of the community of such bioburdens but may transient populations or environmental contaminants. These large numbers are also strongly influenced by the very low relative abundance populations, which for the sake of discussion might be considered populations occurring at $<0.1 \%$ in wounds, yet their numbers still contribute to such diversity estimates. We also observed a relatively high standard deviation in relation to these diversity estimators suggesting that there is a broad range of diversity among pressure ulcers. In addition although we took every preprocessing precaution to enable the most accurate diversity estimation and modeling, newer methods must certainly be tested and validated to provide more accurate descriptions of the microbial diversity in environments such as wounds.

Diversity measurements (Table 3) coordinated with the community structures represented in Figures 1 and Figure 2 illustrate that in most ulcers there are a handful primary pathogens and opportunistic pathogens that make up the largest components of these communities. Thus, chronic wounds are highly polymicrobial and predominant populations (e.g. $>0.1 \%$ of total proportion) must be considered clinically as potential opportunistic players in such infections. To ignore even a potential role of such microorganisms is also to limit the potential efficacy of any type of therapy that is targeting the microbial bioburden/biofilm. If we target only the primary population then ecological principles of selection will dictate that minor populations will succeed into this, now available, niche. If we can find a combination of treatments (e.g. antibiotic, antibiofilm, or antimicrobial) that can target all of the primary or predominant populations in a wound then we have introduced a potentially larger barrier that the very minor populations must overcome to establish their own niche. Even as new populations potentially integrate into the bioburden a new round of diagnostics and a new targeted therapy can be implemented. This becomes a very easy cycle of diagnose and treat that is the cornerstone of medicine. To further the case and point recent diagnostic developments in relation to chronic wounds and their polymicrobial bioburdens, have already been shown to provide definitive and significant improvement in both healing rate and outcome [20]. Now taking this comprehensive diagnostics to the next level, targeted and personalized therapies directed by these diagnostics are currently available. Clinical effectiveness studies are currently in final stages and already clearly show how personalized treatments directed by comprehensive molecular diagnostics may change the way wounds are treated thereby improving the lives of those afflicted and saving millions if not billions in health care dollars.

\section{Conclusion}

The present study is a continuation of the research studies conducted by Dowd et al. and Wolcott et al. $[29,30,35,37]$ using bTEFAP to survey the microbial ecology of diabetic foot ulcers, venous leg ulcers, surgical site infections, and decubitus ulcers. The overwhelming outcome of these collective studies is that traditional culture techniques are still largely inadequate in determining the microbial composition of many chronic wounds. Chronic infections such as Venous, Diabetic

Table 3 Summary of diversity estimators Rarefaction, Shannon index, Ace, and Chao 1

\begin{tabular}{lrrrrr}
\hline Variable & Observations & Minimum & Maximum & Mean & Std. deviation \\
\hline Rarefaction 3\% & 49 & 117.000 & 601.000 & 337.898 & 107.599 \\
Rarefaction 5\% & 49 & 41.000 & 327.000 & 180.082 & 71.995 \\
Shannon 3\% & 49 & 2.896 & 5.614 & 4.703 & 0.670 \\
Shannon 5\% & 49 & 2.353 & 5.166 & 3.955 & 0.763 \\
OTU 3\% & 49 & 117.000 & 601.000 & 337.898 & 107.599 \\
OTU 5\% & 49 & 41.000 & 327.000 & 180.082 & 71.995 \\
ACE 3\% & 49 & 181.962 & 911.814 & 446.255 & 159.805 \\
ACE 5\% & 49 & 72.165 & 515.946 & 235.668 & 102.497 \\
Chao1 3\% & 49 & 189.571 & 733.412 & 436.274 & 145.754 \\
Chao1 5\% & 49 & 72.000 & 412.263 & 228.350 & 94.776 \\
\hline
\end{tabular}

Diversity metrics related to $3 \%$ and $5 \%$ divergence (species and genera levels respectively) are provided along with basic summary statistics. These include the minimum values across all samples, the maximum, the mean and the standard deviation. 
extremity, and Pressure ulcers, have proven to be much more of a diverse and complicated microbial ecology than previously recognized. Most interestingly, obligate anaerobes were found to be a significant proportion, if not a clear majority in chronic wounds, in all surveyed wound types. Requiring specialized collection, transport, and analysis methods for culture based diagnostics of anaerobes will remain difficult especially as part of polymicrobial infections. We demonstrate that chronic wounds such as decubitus ulcers are not merely infected by a single pathogenic species of bacteria, but rather by a blending of symbiotic microbes which form a CWPB. Whether termed bioburden or biofilm, the microbial participation in chronic wounds have been shown to represent a major contributing factor to the resistance of natural healing in chronic wounds, $[37,49,50]$ and the great diversity of these microbial communities adds to their resilience against traditional host and medicinal onslaughts [32]. Previous efforts along with the present study indicate that the highly unique profile of each individual wound would require a therapeutic approach specifically tailored to the patient's respective wound microflora in addition to the multitude of procedures already implemented in the assuage of chronic wounds and support of the host and comorbidities [51,52].

\section{Methods}

\section{General sample collection methods}

Patients were identified with decubitus ulcers and then enrolled in the study protocol after being educated and signing the informed consent protocol in compliance with Western Institutional Review Board approved protocols 56-RW-004 WIRB Protocol \#20062347. All necessary details were thoroughly explained to the patients and written consent was obtained in the presence of a third party witness. A copy of the consent form has been provided to the journal editors. Patients were knowledgeable of their right to discontinue participation at any time despite the established written consent. Samples from decubitus ulcer wound-beds were collected using sharp debridement as a regimen of standard care with aseptic precautions. Samples were placed into sterile $2 \mathrm{ml}$ eppendorf tubes and frozen at $-80^{\circ} \mathrm{C}$. For metadata analysis we utilized age groups 20 (20-40), 50 (41-60), 80 (61 and older), presence of vascular or heart problems, diabetes, systemic antibiotic use, number of days with wounds at time of evaluation (arbitrarily grouped into $<100$ days, $100-900$, and $901+$ days), presence or absence of any type of immobility (e.g. paraplegia or quadriplegia), race, and gender.

\section{DNA Extraction}

After thawing, the debridement samples were centrifuged at 14,000 rpm for 30 seconds and resuspended in
$500 \mu$ l RLT buffer (Qiagen, Valencia, CA) (with $\beta$-mercaptoethanol). A sterile $5 \mathrm{~mm}$ steel bead (Qiagen, Valencia, CA) and $500 \mu \mathrm{l}$ sterile $0.1 \mathrm{~mm}$ glass beads (Scientific Industries, Inc., NY, USA) were added for complete bacterial lyses in a Qiagen TissueLyser (Qiagen, Valencia, $\mathrm{CA}$ ), run at $30 \mathrm{~Hz}$ for $5 \mathrm{~min}$. Samples were centrifuged briefly and $100 \mu \mathrm{l}$ of $100 \%$ ethanol added to a $100 \mu \mathrm{l}$ aliquot of the sample supernatant. This mixture was added to a DNA spin column, and DNA recovery protocols were followed as instructed in the QIAamp DNA Mini Kit (Qiagen, Valencia, CA) starting at step 5 of the Tissue Protocol. DNA was eluted from the column with 30 $\mu \mathrm{l}$ water and samples were diluted accordingly to a final concentration of $20 \mathrm{ng} / \mu \mathrm{l}$. DNA samples were quantified using a Nanodrop spectrophotometer (Nyxor Biotech, Paris, France).

\section{Massively parallel bTEFAP and bTEFAP titanium}

Bacterial tag-encoded FLX amplicon pyrosequencing (bTEFAP) was performed as described previously [37]. The new bacterial tag-encoded FLX-Titanium amplicon pyrosequencing (bTEFAP) approach is based upon similar principles to the previous generation of BTEFAP but utilizes Titanium reagents and titanium procedures and a one-step PCR, mixture of Hot Start and HotStar high fidelity taq polymerases, and amplicons originating from the $27 \mathrm{~F}$ region numbered in relation to $E$. coli rRNA. The bTEFAP procedures were performed at the Research and Testing Laboratory (RTL; Lubbock, TX) based upon RTL protocols http://www.researchandtesting.com.

\section{Bacterial diversity data analysis}

Following sequencing, all failed sequence reads, low quality sequence ends and tags were removed. Sequences were depleted of any non-bacterial ribosome sequences and definite chimeras using custom software described previously [53] and the Black Box Chimera Check software B2C2 (described and freely available at http://www.researchandtesting.com/B2C2. html. Sequences less than $350 \mathrm{bp}$ (bTEFAP titanium) were removed. The identities of decubitus ulcer sequences were determined by first using a distributed BLASTn .NET algorithm [54] against a database of high quality $16 \mathrm{~s}$ bacterial sequences derived from NCBI. Database sequences were characterized as high quality based upon the criteria of RDP ver 9 [55] Using a .NET and C\# analysis pipeline the resulting BLASTn outputs were compiled, validated using taxonomic distance alignment methods, and data reduction analysis performed as described previously $[30,37,56]$. Rarefaction, ace and Chao 1 to estimate maximum diversity in wound using of $300 \mathrm{bp}$ trimmed, nonribosomal sequence depleted, chimera depleted, high 
quality reads was performed as described previously [53,56,29,30] using Mothur [57-59].

\section{Bacterial identification}

The bacteria were classified at the appropriate taxonomic levels based upon the above BLASTn derived sequence identity (percent of total length query sequence which aligns with a given database sequence) and validated using taxonomic distance methods. Sequences with identity scores, to known or well characterized $16 \mathrm{~S}$ sequences, greater than $97 \%$ identity $(<3 \%$ divergence) were resolved to the species, between $95 \%$ and $97 \%$ to the genus, between $90 \%$ and $95 \%$ to the family and between $80 \%$ and $90 \%$ to the order level etc. After resolving based upon these parameters, the percentage of each bacterial ID was individually analyzed for each wound providing relative abundance information within and among the decubitus ulcers based upon relative numbers of reads within a given sample. Evaluations presented at a given taxonomic level, except specific level, represent all sequences resolved to their primary generic identification or their closest relative (where indicated).

\section{Basic Statistics}

Statistics were performed using comparative functions and multivariate hierarchical clustering methods of NCSS 2007 (NCSS, Kaysville, Utah). Using methods described previously, a Unifrac-based principle component analysis (PCA) was utilized to determine significant relationships in the microbial population of ulcers based upon metadata. In short sequences were trimmed to include a uniform length of 350 assembled at 99\% similarity using NGEN (DNAstar, Madison WI), alignment of unique sequences performed with Muscle [58], distance matrix created using DNAdist algorithm of the Phylip package [59], and PCA analysis performed in R (R Development Core Team). Other basic statistics were performed in excel and with XLStat (Addinsoft, NY)

\section{Author details \\ ${ }^{1}$ Research and Testing Laboratory, Lubbock, TX 79407, USA. ${ }^{2}$ Southwest Regional Wound Care Center, Lubbock, TX 79410, USA. ${ }^{3}$ Medical Biofilm} Research Institute, Lubbock, TX 79407, USA.

\section{Authors' contributions}

DMS, DS, JDH prepared and edited manuscript, AMZ, YS, JW performed laboratory methods, RDW obtained clinical samples, SK assisted with data analysis, SED conceived of project, developed molecular and analysis tools, analyzed data, reviewed final versions of manuscripts. All authors have read and approved the final manuscript.

\section{Competing interests}

SED and RDW are owners of Research and Testing Laboratory which provides commercial and research services related to bTEFAP. SED and RDW are owners of Pathogenius Diagnostics which is a clinical diagnostic company with a specific focus on diagnosis of chronic wound infections.
SED and RDW have patents submitted on molecular pathogen diagnostic methods. All other authors are employees of either Pathogenius Laboratory or Research and Testing Laboratory. SK was a student worker with no formal ties any no competing interests.

Received: 11 April 2010 Accepted: 21 September 2010

Published: 21 September 2010

\section{References}

1. Beckrich K, Aronovitch S: Hospital-acquired pressure ulcers: a comparison of costs in medical vs surgical patients. Nurs Econ 1999, 17:263-271.

2. Zulkowski K, Langemo D, Posthauer M: NPUAP. Coming to Consensus on Deep Tissue Injury. Advances in Skin and Wound Care 2005, 18:28-29.

3. Thomson-Rawling J: Pathological Change in Mummies. Proc $R$ Soc Med 1961, 54:409-415.

4. Bansal C, Scott R, Stewart D, Cockerell CJ: Decubitus ulcers: A review of the literature. International journal of dermatology 2005, 44:805.

5. Gefen A: Risk factors for a pressure related deep tissue injury: a theoretical model. Medical and Biological Engineering and Computing 2007, 45:563-573.

6. Gelis A, Dupeyron A, Legros P, Benaim C, Pelissier J, Fattal C: Pressure ulcer risk factors in persons with $\mathrm{SCl}$ : part I: acute and rehabilitation stages. Spinal Cord 2009, 47:99-107.

7. Sakai K, Sanada H, Matsui N, Nakagami G, Sugama J, Komiyama C, et al: Continuous monitoring of interface pressure distribution in intensive care patients for pressure ulcer prevention. 3rd Congress of the WorldUnion-of-Wound-Healing-Societies, Montreal, CANADA 2008, 809-817.

8. Ebright JR: Microbiology of chronic leg and pressure ulcers: Clinical significance and implications for treatment. Nursing Clinics of North America 2005, 40:207-216.

9. Vermillion C: Operating room acquired pressure ulcers. Plastic Surgical Nursing 1990, 10:181.

10. Reddy M, Gill SS, Kalkar SR, Wu W, Anderson PJ, Rochon PA: Treatment of Pressure Ulcers A Systematic Review. Jama-Journal of the American Medical Association 2008, 300:2647-2662.

11. Fowler EC, May WC, Wysocki AB: Decubitus Ulcers in the Do-NotResuscitate Population: Risk Factors and Comorbidities. Wound Repair and Regeneration 2009, 17:A56.

12. Guihan M, Garber SL, Bombardier CH, Goldstein B, Holmes SA, Cao L: Predictors of Pressure Ulcer Recurrence in Veterans With Spinal Cord Injury. Journal of Spinal Cord Medicine 2008, 31:551-559.

13. Brook I: Anaerobic and aerobic bacteriology of decubitus ulcers in children. The American Surgeon 1980, 46:624.

14. Zollo MB, Gostisha ML, Berens RJ, Schmidt JE, Weigle CGM: Altered skin integrity in children admitted to a pediatric intensive care unit. Journal of Nursing Care Quality 1996, 11:62-67.

15. Curley MAQ, Quigley SM, Lin M: Pressure ulcers in pediatric intensive care: Incidence and associated factors. Pediatric Critical Care Medicine 2003, 4:284.

16. Vohra RK, McCollum CN: Pressure Sores. BMJ 1994, 309:853-857.

17. Macklebust J: Pressure ulcers: etiology and prevention. Nursing Clinics of North America 1987, 22:359-377.

18. Russell L: Physiology of the skin and prevention of pressure ulcers. British Journal of Sports Medicine 1998, 7:1084-1100.

19. Haleem S, Heinert G, Parker MJ: Pressure sores and hip fractures. International Journal of the Care of the Injured 2008, 39:219-223.

20. Wolcott R, Ehrlich GD: Biofilms and chronic infections. Jama-Journal of the American Medical Association 2008, 299:2682-2684.

21. Howell-Jones RS, Wilson MJ, Hill KE, Howard AJ, Price PE, Thomas DW: A review of the microbiology, antibiotic usage and resistance in chronic skin wounds. Journal of Antimicrobial Chemotherapy 2005, 55:143-149.

22. James GA, Swogger E, Wolcott R, Pulcini ED, Secor P, Sestrich J, Costerton JW, Stewart PS: Biofilms in chronic wounds. Wound Repair and Regeneration 2008, 16:37-44.

23. Frank DN, Wysocki A, Specht-Glick DD, Rooney A, Feldman RA, St Amand AL, Pace NR, Trent JD: Microbial diversity in chronic open wounds. Wound Repair and Regeneration 2009, 17:163-172.

24. Edwards R, Harding KG: Bacteria and wound healing. Current Opinion in Infectious Diseases 2004, 17:91-96.

25. Donlan RM, Costerton JW: Biofilms: Survival mechanisms of clinically relevant microorganisms. Clinical Microbiology Reviews 2002, 15:167-+ 
26. Costerton JW, Greenberg EP: Bacterial biofilms: A common cause of persistant infections. Science 1999, 284:1318-1322.

27. Ehrlich GD, Hu FZ, Shen K, Stoodley P, Post JC: Bacterial Plurality as a General Mechanism Driving Persistence in Chronic Infections. Clin Orthop Relat Res 2005, 437:20-24.

28. Hill KE, Davies CE, Wilson MJ, Stephens P, Harding KG, Thomas DW: Molecular analysis of the microflora in chronic venous leg ulceration. $J$ Med Microbiol 2003, 52:365-369.

29. Wolcott RD, Gontcharova V, Sun Y, Dowd SE: Evaluation of the bacterial diversity among and within individual venous leg ulcers using bacterial tag-encoded FLX and Titanium amplicon pyrosequencing and metagenomic approaches. BMC Microbiology 2009, 9:226-237.

30. Wolcott RD, Gontcharova V, Sun Y, Zischkau AM, Dowd SE: Bacterial diversity in surgical site infections: not just aerobic cocci any more Journal of Wound Care 2009, 18:103-104.

31. Ebright JR: Microbiology of chronic leg and pressure ulcers: Clinical significance and implications for treatment. Nursing Clinics of North America 2005, 40:207-216.

32. Boles BR, Thoendel M, Singh PK: Self-generated diversity produces "insurance effects" in biofilm communities. Proceedings of the National Academy of Sciences USA 2004, 101:16630-16635.

33. Sun Y, Dowd SE, Smith E, Rhoads DD, Wolcott RD: In vitro multi species Lubbock chronic wound biofilm model. Journal of Wound Care 2008, 16:805-813.

34. Leake JL, Dowd SE, Wolcott RD, Zischkau AM: Identification of yeast in wounds using new pathogen-detection techniques. Journal of Wound Care 2009, 18:103-104.

35. Dowd SE, Sun Y, Secor PR, Rhoads DD, Wolcott BM, James GA, Wolcott RD: Survey of bacterial diversity in chronic wounds using Pyrosequencing, DGGE, and full ribosome shotgun sequencing. BMC Microbiology 2008, 8:43.

36. Dowd SE, Wolcott RD: Molecular Diagnostics to Improve Therapy Choice and Enhance Wound Care. Wound Repair and Regeneration 2009, 17:A32.

37. Dowd SE, Wolcott RD, Sun Y, McKeehan T, Smith E, Rhoads D: Polymicrobial nature of chronic diabetic foot ulcer biofilm infections determined using bacterial tag encoded FLX amplicon pyrosequencing (bTEFAP). PLOS ONE 2008, 3:e3326.

38. Dufort S, Richard M-J, de Fraipont F: Pyrosequencing method to detect KRAS mutation in formalin-fixed and paraffin-embedded tumor tissue. Analytical Biochemistry 2009, 391:166-168.

39. Kennedy JP, Jones CE, Dowd SE: Biofilm Formation Inhibition by Sanguitec (R) Engineered Dressing Systems in a Chronic Wound Biofilm Model. Wound Repair and Regeneration 2009, 17:A33.

40. Reddy M, Gill SS, Kalkar SR, Wu W, Anderson PJ, Rochon PA: Treatment of Pressure Ulcers A Systematic Review. Jama-Journal of the American Medical Association 2008, 300:2647-2662.

41. Davies CE, Hill KE, Newcombe RG, Stephens P, Wilson MJ, Harding KG, Thomas DW: A prospective study of the microbiology of chronic venous leg ulcers to reevaluate the clinical predictive value of tissue biopsies and swabs. Wound Repair and Regeneration 2007, 15:17-22

42. Stackebrandt E, Goebel BM: A place for DNA-DNA reassociation and $16 \mathrm{~S}$ ribosomal-RNA sequence-analysis in the present species definition in bacteriology. Int J Syst Bacteriol 1994, 44:846-849.

43. Gao Z, Tseng C, Pei Z, Blaser MJ: Molecular analysis of human forearm superficial skin bacterial biota. Proc Natl Acad Sci USA 2007, 104:2927-2932.

44. Fierer $\mathrm{N}$, Hamady $\mathrm{M}$, Lauber $\mathrm{CL}$, Knight R: The influence of sex handedness, and washing on the diversity of hand surface bacteria. Proc Natl Acad Sci USA 2008, 105:17994-17999.

45. Grice EA, Kong HH, Conlan S, Deming CB, Davis J, Young AC, Bouffard GG, Blakesley RW, Murray PR, Green ED, Turner ML, Segre JA: Topographical and temporal diversity of the human skin microbiome. Science 2009, 324:1190-1192.

46. Grice EA, Kong HH, Renaud G, Young AC, Bouffard GG, Blakesley RW, Wolfsberg TG, Turner ML, Segre JA: A diversity profile of the human skin microbiota. Genome Res 2008, 18:1043-1050.

47. Roth RR, James WD: Microbial ecology of the skin. Annu Rev Microbiol $1988,42 \cdot 441-464$

48. Cogen AL, Nizet V, Gallo RL: Skin microbiota: a source of disease or defense? Br J Dermatol 2008, 158(3):442-455.
49. Wolcott RD, Rhoads DD, Dowd SE: Biofilms and chronic wound inflammation. Journal of Wound Care 2008, 17:333-341.

50. Bjarnsholt T, Kirketerp-Moller K, Jensen PO, Madsen KG, Phipps R, Krogfelt K, Hoiby N, Givskov M: Why chronic wounds will not heal: a novel hypothesis. Wound Repair and Regeneration 2008, 16:2-10.

51. Wolcott RD, Rhoads DD: A study of biofilm-based wound management in subjects with critical limb ischaemia. Journal of Wound Care 2008, 17:145-155.

52. Wolcott RD, Cox SB, Dowd SE: Healing and healing rates of chronic wounds in the age of molecular pathogen diagnostics. J Wound Care 2010, 19:272-1.

53. Dowd SE, Callaway TR, Wolcott RD, Sun Y, McKeehan T, Hagevoort RG, Edrington TS: Evaluation of the bacterial diversity in the feces of cattle using $16 \mathrm{~S}$ rDNA bacterial tag-encoded FLX amplicon pyrosequencing (bTEFAP). BMC Microbiology 2008, 8:125.

54. Dowd SE, Zaragoza J, Rodriquez JR, Oliver MJ, Payton PR: Windows .NET Network Distributed Basic Local Alignment Search Toolkit (W.ND-BLAST). BMC Bioinformatics 2005, 6:93.

55. Cole JR, Wang Q, Cardenas E, Fish J, Chai B, Farris RJ, Kulam-SyedMohideen AS, McGarrell DM, Marsh T, Garrity GM, Tiedje JM: The Ribosomal Database Project: improved alignments and new tools for rRNA analysis. Nucleic Asids Res 2009, 37:D141-D145.

56. Acosta-Martinez V, Dowd SE, Sun Y, Allen V: Tag-encoded pyrosequencing analysis of bacterial diversity in a single soil type as affected by management and land use. Soil Biology and Biochemistry 2009, 4:2770.

57. Schloss PD, Westcott SL, Ryabin T, Hall JR, Hartmann M, Hollister EB, et al: Introducing mothur: open-source, platform-independent, communitysupported software for describing and comparing microbial communities. Appl Environ Microbiol 2009, 75:7537-7541.

58. Edgar RC: MUSCLE: multiple sequence alignment with high accuracy and high throughput. Nucleic Acids Res 2004, 32:1792-1797.

59. Retief JD: Phylogenetic analysis using PHYLIP. Methods Mol Biol 2000 132:243-258

\section{Pre-publication history}

The pre-publication history for this paper can be accessed here: http://www.biomedcentral.com/1755-8794/3/41/prepub

doi:10.1186/1755-8794-3-41

Cite this article as: Smith et al:: Evaluation of the bacterial diversity of Pressure ulcers using bTEFAP pyrosequencing. BMC Medical Genomics 2010 3:41.

\section{Submit your next manuscript to BioMed Central and take full advantage of:}

- Convenient online submission

- Thorough peer review

- No space constraints or color figure charges

- Immediate publication on acceptance

- Inclusion in PubMed, CAS, Scopus and Google Scholar

- Research which is freely available for redistribution

Submit your manuscript at www biomedcentral com/submit
Biomed Central 